\title{
Fatal right ventricular infarction caused by Bioglue coronary embolism
}

\author{
Zahid Mahmood, FRCS, ${ }^{a}$ Debbie S. Cook, MRCPath, ${ }^{b}$ Heyman Luckraz, FRCS, ${ }^{a}$ and Peter O'Keefe, FRCS (CTh), \\ Cardiff, United Kingdom
}

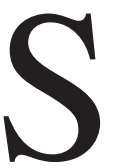

ince the experimental work of Koehnlein and Lemperle ${ }^{1}$ on the use of glue in operations, biologic glues have become part of the armamentarium used in the surgical management of patients undergoing repair of type A aortic dissection, especially where tissues are frail or where added hemostasis is required. It facilitates the reconstruction of the dissected aortic wall layers adjacent to the prosthetic graft. However, the use of tissue glue can be associated with significant risk, namely aortic wall necrosis leading to acute aortic redissection, ${ }^{2}$ coronary ostial stenosis, cerebral embolism, ${ }^{3}$ complete heart block, and prosthetic valvular dysfunction. We describe a case in which the glue embolized to the right and left coronary arteries, leading to fatal right ventricular infarction, and also demonstrate the usefulness of postmortem studies with the use of digital technologies for records keeping and educational purposes.

\section{Clinical Summary}

A 74-year-old woman was referred to our care for emergency repair of type A aortic dissection. The procedure was carried out with deep hypothermic circulatory arrest. The operative findings included a morphologically normal aortic valve with mobile cusps and the proximal extent of the dissection occurring within the noncoronary sinus, with detachment of the noncoronary-right coronary commissure. A tear was also identified on the anteromedial aspect of the proximal arch adjacent to the orifice of the brachiocephalic artery. The false lumen was seen progressing beyond the arch, involving both its convexity and concavity.

The aortic valve was repaired with resuspension of the cusps. Bioglue (Cryolife Inc, Kennesaw, Ga) was injected in the false lumen in the noncoronary sinus and around the false lumen at the level of the sinotubular junction. The hemiarch was replaced with a 24-mm Hemashield gold vascular graft (Boston Scientific Meditech, Boston, Mass). Bioglue was inserted within the false lumen at the arch level, and the aortic layers were sealed together. After rewarming the patient, cardiopulmonary bypass was discontinued, with good hemodynamic parameters on $5 \mu \mathrm{g} \cdot \mathrm{kg}^{-1} \cdot \min ^{-1}$ of dopamine.

\footnotetext{
From the Cardiothoracic Unit ${ }^{\mathrm{a}}$ and Wales Institute of Forensic Medicine, ${ }^{\mathrm{b}}$ University Hospital of Wales, Heath Park, Cardiff, United Kingdom.

Received for publication March 31, 2004; accepted for publication April 6, 2004.

Address for reprints: Heyman Luckraz, FRCS, Cardiothoracic Unit, Block C5, University Hospital of Wales, Heath Park, Cardiff CF14 4XN, United Kingdom (E-mail: HeymanLuckraz@aol.com).

J Thorac Cardiovasc Surg 2004;128:770-1

$0022-5223 / \$ 30.00$

Copyright $\odot 2004$ by The American Association for Thoracic Surgery

doi:10.1016/j.jtcvs.2004.04.033
}

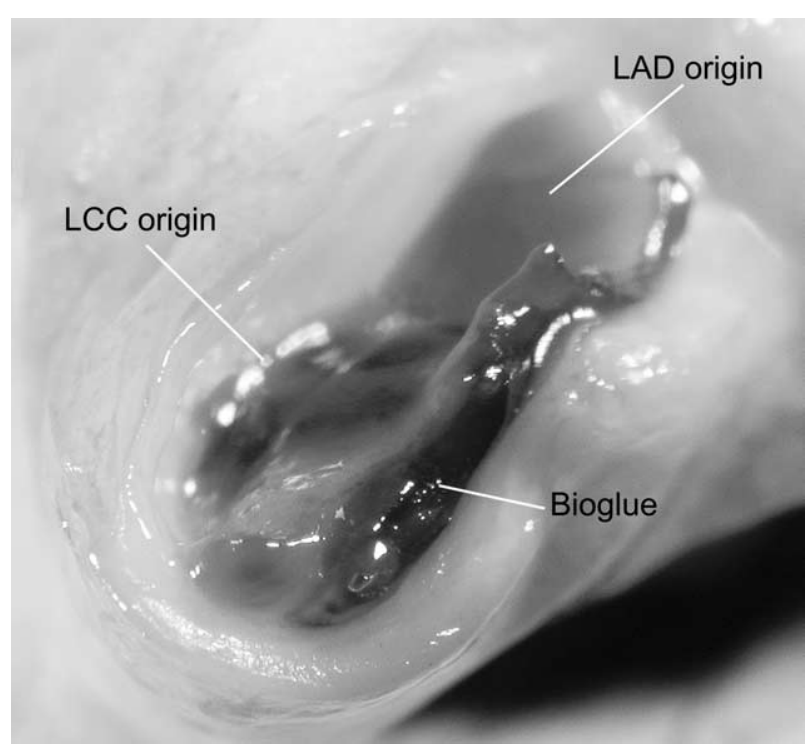

Figure 1. Occlusion of the origin of the left circumflex artery caused by the Bioglue with distal propagated thrombus. LCC, Left circumflex coronary artery; $L A D$, left anterior descending coronary artery.

The patient made a slow but steady recovery postoperatively until the sixth postoperative day, when she had an episode of chest pain associated with being cold and clammy. She was tachycardic, tachypnoeic, and hypotensive, with electrocardiography showing signs of inferior infarction. An echocardiogram showed a significant pericardial collection, right ventricular akinesia, and moderate mitral regurgitation. She underwent emergency resternotomy, which confirmed $500 \mathrm{~mL}$ of bloody pericardial effusion and a distended, akinetic right ventricle. She did not respond to inotropic support, and resuscitative measures were abandoned.

A postmortem examination was requested, with consent for a limited examination of the heart and aorta and no retention of tissues or organs. This showed pale and soft areas within the right ventricular myocardium with mottled, hemorrhagic zones. Further macroscopic examination of the coronary tree revealed occlusion of the origin of the left circumflex artery caused by the Bioglue with distal propagated thrombus (Figure 1). Bioglue emboli were also identified within the right coronary artery and the diagonal branch of the left anterior descending artery. Bioglue from the patient's coronary arteries was compared with freshly prepared Bioglue from the glue tube by means of dissecting microscopy. This examination revealed that they were identical in appearance (amber, translucent, agar-like, solid fragments with crisp margins). 


\section{Discussion}

Several biologic sealants are now available for use in cardiovascular surgery, with the best studied being gelatin-resorcin-formalin (GRF) glue and a 2-component fibrin sealant. The use of GRF glue has been associated with tissue necrosis, with destruction of the aortic media causing vascular wall thinning and rupture. ${ }^{2}$ Its use has also been linked with coronary ostial stenosis and fatal cerebral embolism. ${ }^{3}$ Bioglue, which is composed of bovine serum albumin (45\%) and gluteraldehyde (10\%), is marketed as being less tissue toxic than GRF (gluteraldehyde 37\%), and initial clinical experience was reported by Raanani and colleagues ${ }^{4}$ in 2001 . Polymerization occurs when the 2 components are mixed, reaching bonding strength within 2 minutes. This tissue glue is also described as being more liquid in consistency than the GRF glue. Its potential to penetrate the intravascular circuit is thus higher. Thus it has the potential to embolize, as in the case described. The emboli then activates the clotting cascade directly and also produces stasis, which is one of the triad described by Virchow necessary for thrombus formation and propagation.
This case also illustrates the importance of the use of postmortem studies to elucidate the cause of death, as well as the use of digital imaging by the pathologists, to demonstrate the postmortem findings, obviating the need for tissue retention. ${ }^{5}$

\section{References}

1. Koehnlein HE, Lemperle G. Experimental studies with a new gelatinresorcin-formaldehyde glue. Surgery. 1969;66(2):377-82

2. Fukunaga S, Karck M, Harringer W, Cremer J, Rhein C, Haverich A. The use of gelatin-resorcin-formalin glue in acute aortic dissection type A. Eur J Cardiothorac Surg. 1999;15:564-70.

3. Mastroroberto P, Chello M. Embolization of biological glue after repair of acute aortic dissection. Ann Thorac Surg. 1996;62:946-7.

4. Raanani E, Latter DA, Errett LE, Bonneau DB, Leclerc Y, Salasidis GC. Use of 'bioglue' in aortic surgical repair. Ann Thorac Surg. 2001;72: $638-40$.

5. Guidelines on autopsy practice. Report of the working group of the Royal College of Pathologists, September 2002. www.rcpath.org.

\title{
A technique for repair of mitral paravalvular leak
}

\author{
Abeel A. Mangi, MD, and David F. Torchiana, MD, Boston, Mass
}

$\mathrm{T}$ he incidence of paravalvular leak 15 years after mitral valve replacement (MVR) approximates 17\%. ${ }^{1,2}$ Its relative incidence after mechanical and bioprosthetic replacement is debated. ${ }^{1}$ Running monofilament suture techniques $^{3}$ and MVR for endocarditis ${ }^{4}$ have been implicated in the development of paravalvular leaks. Twenty-two percent of patients with paravalvular leaks are diagnosed in the first week after MVR, and another 52\% are diagnosed within the first postoperative year. Patients requiring operative repair are older, have symptomatic heart failure, hemolytic anemia, and larger leaks. Surgical intervention to repair the leak improves symptoms of congestive heart failure, augments the hematocrit value, decreases the need for blood transfusion, and is an independent predictor of long-term survival when compared with medical therapy. ${ }^{4}$ Choice of operation involves either direct suture repair of the leak site, which carries a failure rate of $13 \%$; or replacement of the valve, which carries a failure rate of up to $35 \%$ and poses a technical

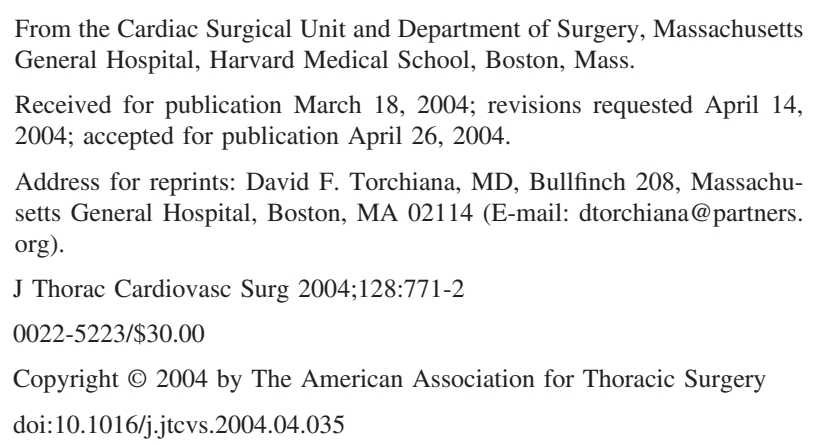

challenge. Here we propose a simple technique for repair of selected mitral paravalvular leaks that incorporates healthy, fullthickness autologous tissue into the repair and has promising durability.

\section{Technique}

The operation is conducted during continuous intraoperative transesophageal echocardiography (TEE) and commences with standard ascending aortic and bicaval cannulation. After the heart is arrested with antegrade intermittent cold blood cardioplegia, a standard superior septal incision is made. If both atria are significantly enlarged, the prosthesis can be approached solely through the interatrial septum. This approach yields excellent exposure of the anterior part of the mitral prosthesis that abuts the interatrial septum and is proximate to the posteromedial commissure of the native mitral valve. In our experience this location accounts for $38 \%$ of mitral paravalvular leaks. ${ }^{5}$ Although TEE accurately identifies $88 \%$ of mitral paravalvular leaks, ${ }^{5}$ clear communication between the surgeon and echocardiographer is essential to ensure that the location and number of leaks are accurately defined, especially because nomenclature in this area can be confusing. As an example, the location that we are discussing is on the anterior aspect of the mitral prosthesis but also is proximate to the posteromedial commissure of the native mitral valve. It is therefore critical that the echocardiographer and the surgeon refer to standardized reference points. Depending on the extent of the leak, the first pledget-supported, braided polyester stitch is placed as a horizontal mattress stitch through a fold of the left atrial wall and is then brought directly into the sewing ring at the most posterior aspect of the leak near the coronary sinus. The remaining sutures 